\title{
Interaction between Time Dependent Exposure Strategies and Part Positioning within Selective Laser Melting Process of Plastics
}

\author{
http://dx.doi.org/10.3991/ijes.v3i1.4286 \\ D. Drummer, M. Drexler, K.Wudy \\ Friedrich-Alexander-University Erlangen-Nürnberg, Germany
}

\begin{abstract}
The selective laser melting of polymer powder is, for rapid prototyping applications, a well-established technology, although a lack in basic process knowledge occurs. Considering demands of series production the selective laser melting technique of polymers is faced with various challenges concerning processable material systems, process strategies and part properties. Consequently, basic research is necessary to understand and optimize processes to shift from rapid prototyping to rapid manufacturing of small lot sized series. Based on basic research the high potential of selective laser melting for the production of complex parts without any tools can be opened up. For the derivation of part quality increasing process strategies, knowledge about interactions between sub-processes of selective laser melting and resulting part properties is necessary. The selective laser melting of polymers consists of three major subprocesses: Powder coating, energy input, material consolidation. According to the interaction of sub-processes, resulting temperature fields during the selective laser melting process determine the part properties by changing micro structural pore number and distribution. Beneath absolute temperatures the time-dependency of the thermal fields also influences the porosity of molten parts. Present process strategies tend to decrease building time by increasing scanning speed and laser power. Although the absolute energy input into the material is constant for increasing scanning speed and laser power in the same ratio, time dependent material effects are neglected. The heating rate is a combined parameter derived from absolute temperature and time. Within the paper the authors analyze the basic interactions between different heating rates and part properties (e. g. mechanical strengths). Furthermore, the part positioning is taken into account. Due to the part positioning within the building chamber different shapes of cross sections appear even for equal part geometries. The authors estimate an interaction between exposed cross section and applied speed of energy input, due to heat accumulating effects. Therefore specimens produced with different heating rates are analyzed with imaging technologies as well as mechanical tests. Based on the done basic investigations new heating rate dependent process strategies can be established considering time dependent material behavior.
\end{abstract}

Index Terms-heating rate, positioning, mechanical properties.

\section{INTRODUCTION}

Formerly, techniques of additive manufacturing were used for prototype construction of special products. This actually changes, as they are now used for a vast number of new application fields by means of direct manufacturing. [1-3]

Especially for technical parts, products made by additive manufacturing have grown in importance, now being much more than mere demonstration objects $[4,5]$. Despite the huge variety of additive manufacturing processes only a few of them have the potential to meet the requirements of small series industrial manufacturing. Considering industrial requirements (e. g. reproducibility, mechanical strength) one of the most promising additive processes is the selective laser melting of semi-crystalline thermoplastic powders (Fig. 1). [6]

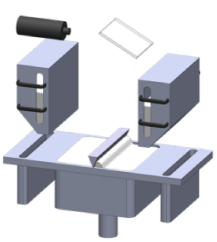

Powder coating

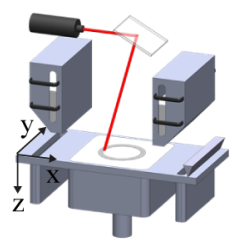

Exposure

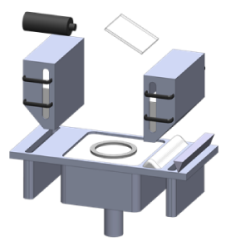

Consolidation
Figure 1. Selective laser melting process (SLM) of thermoplastic polymers

To achieve industrial standards with beam molten thermoplastic parts basic process exploration is essential. For this purpose the sub-processes (powder coating, energy input, material consolidation) of component generation and their mutual interactions (e. g. time dependent material behavior and energy input) are investigated experimentally by a specific combination of different measurement methods. Thus, selective laser sintering can be benchmarked in its entirety. Based on this basic research and built on process modeling, new adapted process strategies for an improved part quality can be developed. Effects like warpage or curl should be reduced. Therefore limited process efficiency can be improved with regard to series production. [7, 8]

In the following article the influence of the length of scanning vectors is analyzed by using mechanical part properties as output quantity. Furthermore, interaction between mechanical part properties and heating rate during exposure (importance of heating rate for part structure was shown in previous investigations by the authors $[9$, 10]) is taken into account. For investigations the statistical design of experiments (DoE) is used. Thus, a basic understanding of the interactions between exposure speed, length of scanning paths and part positioning in $x-y-p l a n e$ is created. 


\section{FUnDAMENTALS AND EXPERIMENTAL SETUP}

\section{A. Laser-Melting-System (LMS)}

To guarantee constant experimental boundary conditions between different experiments, it is necessary to use a special temperature stabilized and homogenized LMS. Due to a multiple heating zone system, the whole building chamber is nearly equally tempered to $\mathrm{T}_{\mathrm{B}}$. Consequently, disturbing effects caused by temperature variation can be minimized. Moreover, a laser system with nearly constant intension over whole building chamber is used (F-Theta-lens). The scanning system is state of the art and guarantees short acceleration times as well as high precision in beam guiding. The focus diameter $d_{f}$ is set to $400 \mu \mathrm{m}$. Due to the very short exposure times within the design of experiments a heating of the optical gating system is avoided. Hence, Gaussian-beam-profile is hardly adulterated for different exposure parameters.

\section{B. Material}

For achieving nearly constant material properties for all experiments, standard laser melting powder, polyamide 12 (PA12) (Type: PA2200, EOS GmbH, Krailling, Germany), is used.

\section{Exposure strategies and specimens}

Usually the exposure process is performed by layers, which are composed out of hatched single lines (Fig. 2).

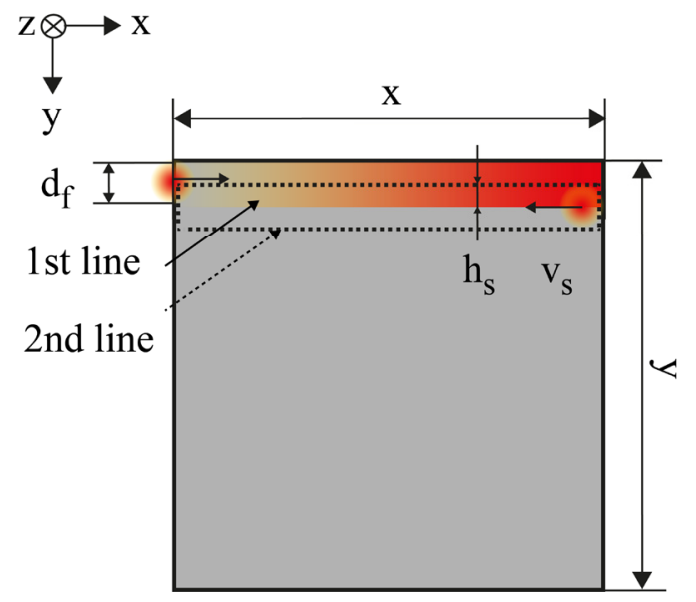

Figure 2. Hatching exposure strategy exemplarily for a plate

After one line is exposed by deflecting the laser beam in $\mathrm{x}$-direction the beam is deflected in revers $\mathrm{x}$-direction, exposing the second line. After each line the beam is also deflected in $\mathrm{y}$-direction over a distance $\mathrm{x}=\mathrm{h}_{\mathrm{s}}$. Due to this $y$-deflection within the hatching strategy two single lines always superpose in the size of $h_{s}$. Consequently, each single line is exposed two times. The time t between two exposures is defined as cooling time. The maximum cooling time (between two exposures of first point of a line) can be approximated by equation 1 . Due to the equation, the cooling time is influenced by scanning speed $\mathrm{v}_{\mathrm{s}}$ as well as path length $\mathrm{x}$ of single lines $\mathrm{n}$ and (n-1).

$$
\mathrm{t}_{\max }=\mathrm{t}_{\mathrm{n}}+\mathrm{t}_{(\mathrm{n}-1)}=\frac{1}{\mathrm{v}_{\mathrm{s}}} \cdot\left(\mathrm{x}_{\mathrm{n}}+\mathrm{x}_{(\mathrm{n}-1)}\right)
$$

Due to the hatching exposure principle the latest trend in construction of LMS is the increase of scanning speed for achieving shorter processing times. Like shown in equation 2 , the impact time for certain laser properties is defined as ratio between beam's focus diameter $d_{f}$ and chosen scanning velocity $\mathrm{v}_{\mathrm{s}}$. Due to the circular shape of beam's cross section the impact time follows trigonometric function. Thus, impact time in beam edge zones is smaller than for the center line.

$$
\mathrm{t}=\frac{\mathrm{d}_{\mathrm{f}}}{\mathrm{v}_{\mathrm{s}}}
$$

Furthermore, by using equation 3 the energy input $E_{\text {in }}$ during the exposure with laser power $\mathrm{P}_{\mathrm{L}}$ can be approximated.

$$
\mathrm{E}_{\mathrm{in}}=\mathrm{P}_{\mathrm{L}} \cdot \mathrm{t}=\mathrm{P}_{\mathrm{L}} \cdot \frac{\mathrm{d}_{\mathrm{f}}}{\mathrm{v}_{\mathrm{s}}}
$$

For achieving a melting of the exposed areas a minimum energy input in the range of the specific melting enthalpy of the used polymer powder is necessary. Related to the optimal thickness of a single layer $(0.1 \mathrm{~mm})$ a common value for used processing energy is $0.4 \mathrm{~J} / \mathrm{mm}^{3}$. Like mentioned before, the combination of high power laser beam sources and high speed laser scanners is used for speeding up the exposure process. Time dependent effects within the processed polymer materials are neglected despite the time dependency of polymer properties is well known in polymer science. Especially the speed of the energy input affects thermal material behavior of the sintering powder, like shown by the authors in [11]. A parameter, which expresses the heating speed is the heating rate $\mathrm{H}$, which is defined as the variation of temperature over a certain space of time (equation 4).

$$
\mathrm{H}=\mathrm{P}_{\mathrm{L}} \cdot \frac{1}{\mathrm{~m} \cdot \mathrm{c}_{\mathrm{p}}}=\frac{\mathrm{dT}}{\mathrm{dt}}
$$

By taking laser power $\mathrm{P}_{\mathrm{L}}$, mass $\mathrm{m}$ of exposed polymer powder as well as the specific heat capacity $c_{p}$ into account, the heating rate in a discrete powder element can be approximated. Based on further explanations even on a constant level of energy input, while hatching single lines, different exposure strategies are possible. On the one hand the energy input can be performed by high laser powers and high scanning speeds (low impact times) and on the other hand low laser powers at moderate scanning speeds can be chosen. The overall done energy input remains equal for both strategies. The authors estimate variations in the microstructure of laser molten parts depending on the used heating rate. Base for this estimation is the wellknown time dependency of thermal polymer properties (e. g. DSC-Analysis) as well as the varying cooling times between two hatching lines according to the scanning speed. Within the hatching principle an exposed line is heated up a second time after exposure due to the exposure of the second line, which overlaps with the first line in the size of the hatching distance $h_{s}$. Hence high heating rates also cause short cooling times between to single lines. Furthermore, the length of a single line also affects cooling time between exposed lines. Overall the so defined cooling conditions interact with the microstructure of the produced parts. Consequently, the authors want to investigate first correlations between speed of energy input as well as length of scanning vectors and mechanical part strength. Tab. 1 shows the investigated exposure parameters. 
TABLE I.

DESIGN OF EXPERIMENTS: EXPOSURE PARAMETERS

\begin{tabular}{cccc}
\hline & \multicolumn{3}{c}{ Process parameters } \\
\hline & Laser-Power $\mathbf{P}_{\mathbf{L}}$ & $\begin{array}{c}\text { Scanning speed } \mathbf{v}_{\mathbf{s}} \\
\left(\text { referenced to } \mathbf{d}_{\mathbf{f}} \text { ) }\right.\end{array}$ & Heating rate $\mathbf{H}$ \\
{$[\mathbf{m m} / \mathbf{s}]$} & {$\left[\mathbf{1 0}^{\mathbf{7}} \cdot \mathbf{K} / \mathbf{m i n}\right]$} \\
\hline 1 & {$[\mathbf{W}]$} & 780 & 7.20 \\
2 & 7.8 & 2210 & 20.40 \\
3 & 22.1 & 3290 & 30.40
\end{tabular}

The laser powers $\mathrm{P}_{\mathrm{L}}$ as well as scanning velocities $\mathrm{v}_{\mathrm{S}}$ of Tab. 1 are orientated on industrial used values and cover a large range of powers and speeds. The varying heating rates are applied during the building process of plate shaped specimen, with following dimensions (Tab. 2).

TABLE II.

DIMENSIONS OF USED SPECIMENS

\begin{tabular}{cccc}
\hline \multicolumn{4}{c}{ Dimensions } \\
\hline $\begin{array}{c}\mathbf{x} \\
{[\mathbf{m m}]}\end{array}$ & $\begin{array}{c}\mathbf{y} \\
{[\mathbf{m m}]}\end{array}$ & $\begin{array}{c}\mathbf{z} \\
{[\mathbf{m m}]}\end{array}$ \\
\hline 1 & 15 & 85 & 2 \\
2 & 30 & 85 & 2 \\
3 & 60 & 85 & 2 \\
4 & 90 & 85 & 2 \\
5 & 85 & 85 & 2
\end{tabular}

First single layers are built to avoid disturbing effects caused by the powder coating mechanism. All single layers are cooled down in the same way after exposure, to avoid influences of changed cooling conditions. The single layers are cut flatwise to the scanning direction and are analyzed by microscopy, like shown in Fig. 3.

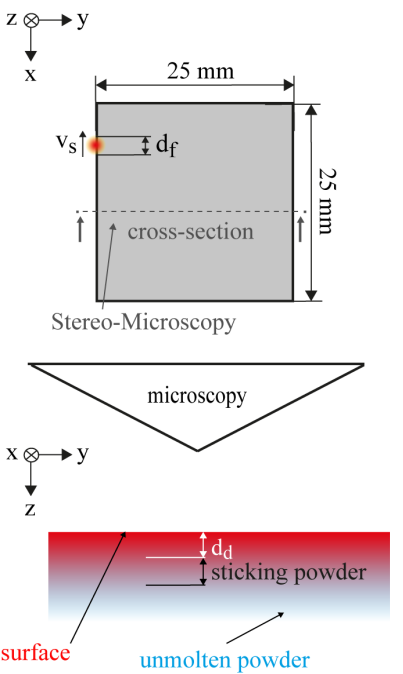

Figure 3. Microscopic analysis of cut single layers

Beneath single layers with constant dimensions layers with a constant surface area but varying widths and lengths are produced.

The hatching distance $h_{s}$ is kept constant over the whole design of experiments $\left(\mathrm{h}_{\mathrm{s}}=250 \mu \mathrm{m}\right)$ and produced specimens. By means of microscopy pictures the geometrical accuracies as well as the sizes of the cross sections of the single layers are judged.

Additionally, size scaled tensile bars (1:2) vertical (x-yplane, " 0 -lying") and parallel (x-z-plane, " $90^{\circ}$-standing") to the $\mathrm{z}$-axis are built with exposure parameters, shown in Tab. 1.
For investigating the influence on the mechanical strength of with different heating rates and path lengths $\mathrm{x}$ exposed parts, flat plates with a thickness of $2 \mathrm{~mm}$ are built. By milling, tensile bars (scaled 1:2 in size according to norm bars) are etched out of the plates in different ways (Fig. 4.)
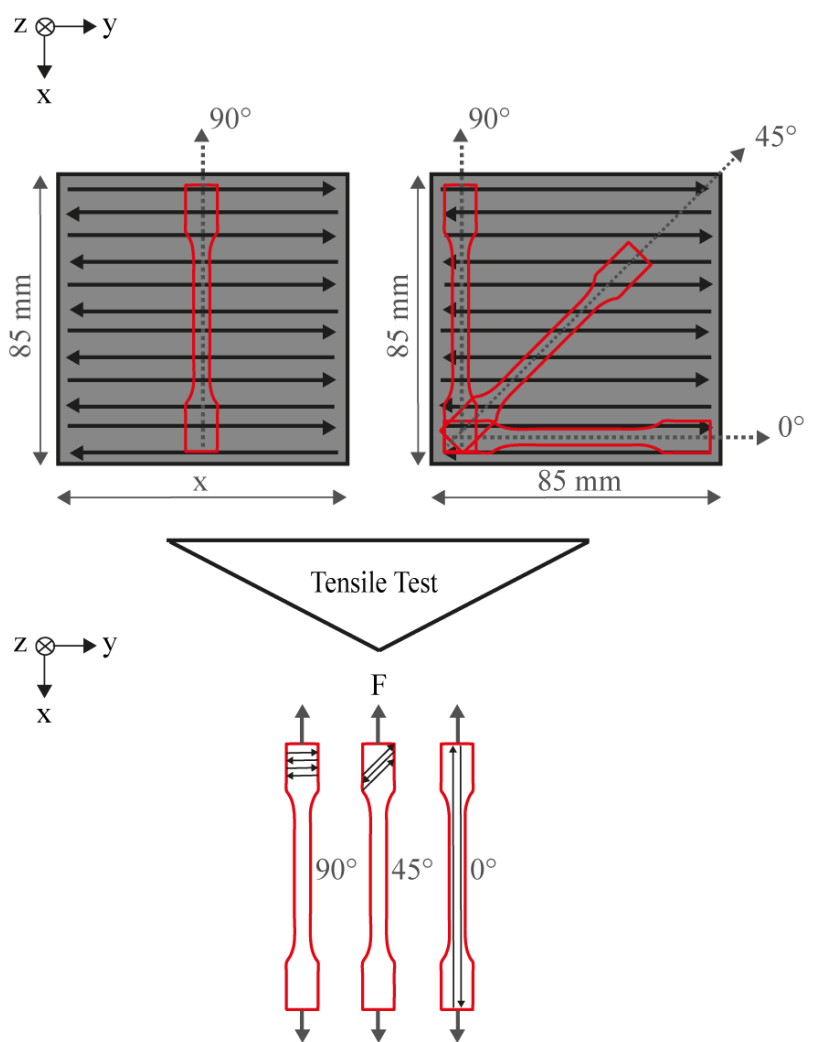

Figure 4. Specimen preparation and testing

The milling process is used for the preparation of tensile bars in order to achieve constant surface roughness between specimens. Thus crack ignition effects caused by varying surface properties can be minimized. Furthermore, by preparing tensile bars in different angles to the scanning vectors out of the plates, the influence of part positioning can be isolated without changing exposure times by varying part positioning. Consequently, by variation of heating rate, length of plate as well as orientation angle between tensile bars and scanning vectors different influences on the mechanical behavior of the tensile bars can be analyzed. Prepared tensile bars are dried under the critical value of 0.2 Weight.- $\%$ of remaining water (method: Karl-Fischer-Titration). Afterwards tensile tests are performed (DIN EN ISO 527-1, -2) with to size scaling adapted speed. Tab. 3 shows test conditions.

TABLE III

TENSILE TEST CONDITIONS

\begin{tabular}{ccc}
\hline & Conditions \\
\hline $\begin{array}{c}\text { Testing Speed } \\
{[\mathrm{mm} / \mathrm{s}]}\end{array}$ & Specimen Conditioning & $\begin{array}{c}\text { Number of tests } \\
{[-]}\end{array}$ \\
\hline $\mathbf{0 , 0 4 2}$ & Dry $(<0.2$ wt.-\% humidity $)$ & 3
\end{tabular}




\section{RESULTS AND DISCUSSION}

\section{A. Single layers}

Fig. 5 shows the cross sections of layers exposed with varying heating rates but constant length of scanning vectors.

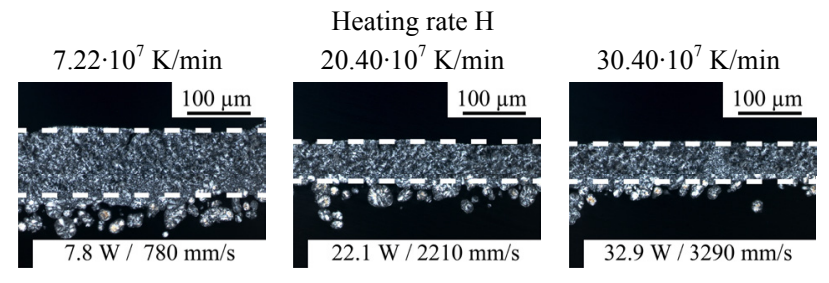

Figure 5. Cross sections of single layers for varying heating rates

Like discussed in further publications of the authors (e. g. $[9,12])$, for an increasing heating rate $\mathrm{H}$, the size of the melting pool (especially z-dimension $d_{d}$, Fig. 6) changes.

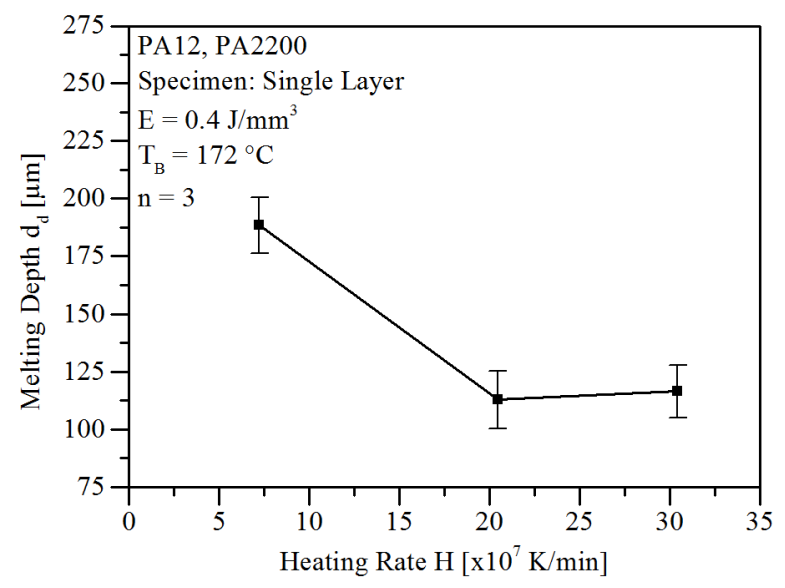

Figure 6. Thickness of with varying heating rates produced layers

For constant length of scanning vectors, the resulting shape of the melt pool is thus a function of the speed of energy input. Reasonable for this correlation might be time dependent material behavior as well as thermodynamics $[9,10]$. Due to varying heating rates $H$ the impact times $t$ for beam-matter-interaction change, too. For larger impact times heat transfer mechanisms into the surrounding powder bed and into the building chamber additionally occurs. Especially heat conduction into the surrounding powder bed is a time-consuming process. Hence, for long impact times and respectively moderate heating rates the amount of conducted heat into the surrounding powder is maximal. A hint for supporting this estimation is the amount of unmelted powder which sticks next to the molten zone as result of the conducted heat out of the melting zone. Especially for further mentioned low heating rates the amount of sticking powder is increased, like shown in $[9,12]$. Considering Fig. 6 higher heating rates seem to favor a heat accumulation next to the exposed surface. Reasonable for this behavior might be the short impact time combined with high laser power. Due to minimized impact times, hardly any time for heat conduction into surrounding powder is availabel. Consequently, absorbed laser energy is distributed only over directly penetrated particles within the optical penetration depth $\delta_{\text {opt }}$ (where most of the intensity is extenuated). Due to this fact particles within the penetration-zone are molten. Based on the fact, that thermal conduction into $\mathrm{z}$-direction is minimized for short impact times a heat accumulation next to the exposed surface occurs [9]. Furthermore, this heat accumulation next to the surface might result in an increased thermal interaction (e. g. irradiation) between exposed area and building chamber. Thus, absorbed laser power is lost and melting depth $d_{d}$ decreases for higher heating rates, like shown in Fig. 6.

Beneath the heating rate also the length of scanning lines influences the structure forming process within the melting zone. Reasonable for this behavior might be also time dependent effects. Due to long scanning paths and constant exposure speeds, the cooling time between double exposure of the same line changes. Consequently, within the hatching exposure strategy the length of the scanning paths are supposed to affect the resulting melt pool shape. Fig. 7 shows the cross section of single layers manufactured with constant heating rate and varying length of scanning vectors. Thus the cooling time between double exposure of single lines changes. Hence thermal dependent material properties (e.g. viscosity, crystallization, post-condensation) might also change.
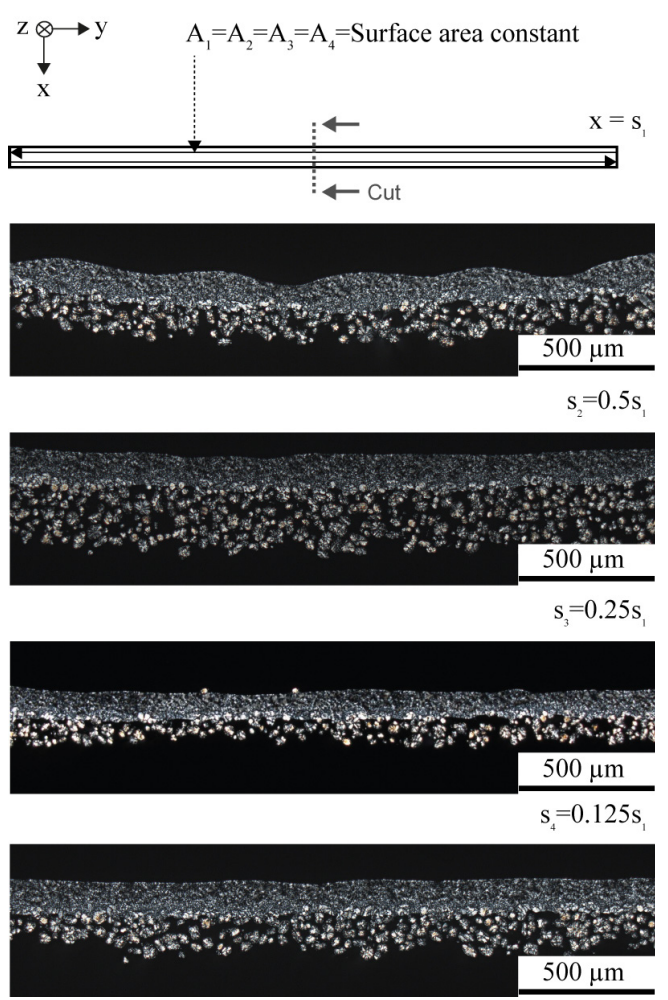

Figure 7. Cross sections of plates for varying scanning-path-length $\mathrm{x}$

It is obvious that for long scanning vectors the shape of the cross section is irregular. Especially the surface of molten layers with long path length $\mathrm{x}$ show a rippled shape of cross section. For reduced length of scanning vectors the ripple of the surface is reduced. By changing length of scanning lines at constant heating rate the cooling times between repeating exposures of single lines are changed. Herein a hint can be seen, that for long scanning vectors in the time between two exposures the melt pool cools down and viscosity of melt pool is reduced lightly. Owing to this fact the fluidity of the melt pool is reduced. Thus two exposed lines are hampered to flow together shaping one melting pool. For decreasing vector length, the time between to exposures declines. 
Hence, the cooling time between exposures is reduced. Thus the fluidity of both exposed lines is preserved and a common melt pool is shaped. The result is a more regular cross section of the single layer. Besides shown effects on single layer level, time dependent effects are also estimated to affect mechanical strength of all parts.

\section{B. Tensile bars}

Fig. 8 shows the tensile strength of a size-scaled (1:2) tensile bar (directly manufactured, without post-treatment) for different heating rates $\mathrm{H}$ as well as orientations to the building platform.

It is visible, that well known mechanical behavior for different orientated tensile bars remains the same for varying heating rates $\mathrm{H}$. The $90^{\circ}$-orientated tensile bars show a reduced tensile strength compared to the $0^{\circ}$-orientated ones, over the whole range of investigated heating rates. Furthermore, for $0^{\circ}$-orientated tensile bars, a trend into direction of decreasing tensile strength for an increased heating rate can be seen. $90^{\circ}$-orientated tensile bars do not show this trend. According elongation at break $\varepsilon$ behave nearly similar to $\sigma$.

Due to an increased heating rate also surface temperature during exposure increases owing to a heat accumulation caused by increasing scanning speeds [9]. In combination with the smaller exposure area of a $90^{\circ}$-orientated tensile bar (compared with a $0^{\circ}$-orientated one) the temperature accumulation for higher heating rates might lead into melt viscosity changes as well as in a cross section increase as a result of a higher temperature flow into the surrounding powder. Thus, the connection between two layers is stronger although melting depth $d_{d}$ of a single layer is reduced for higher heating rates. For $0^{\circ}$-orientated bars the superposition of thermal effects is not as strong because heat accumulation is minored due to larger cooling time between line-hatching because of longer scanning paths.

The influence of path length $\mathrm{x}$ for $\mathrm{x}-\mathrm{y}$-plane-placed $\left(0^{\circ}\right.$ lying) tensile bars can be seen in Fig. 10.

Considering the tensile strength of bars (x-y-plane, $0^{\circ}$ lying) produced with varying lengths of scanning paths $\mathrm{x}$, no significant changes can be investigated. Furthermore, no significant differences in tensile strengths for changed heating rates can be investigated. Based on cross sections (shown in Fig. 7) accuracy of melting pool changes with decreasing length $\mathrm{x}$ of scanning paths. Especially very long scanning paths $\mathrm{x}$ seem to result in irregular shaped cross sections, which seems to abet defects within the cross section. Consequently, the authors estimated reduced tensile strength for increased path length. Reasonable for the ambiguous behavior of tensile properties, shown in Fig. 10, might be the superposition of several layers. Hence, small irregularities and defects within the layer might be normalized by the layer manufactured upon. Due to this fact, tensile behavior even for long scan paths does not change significantly. Considering the elongations at break, two trends might be visible. On the one hand for constant heating rates the elongation at break seems to decrease for enlarged scanning paths. Especially for small heating rates $\mathrm{H}$ prior described trend can be seen in Fig. 11.

On the other hand by increasing heating rates, the elongations at break decreases. Additionally, elongations for

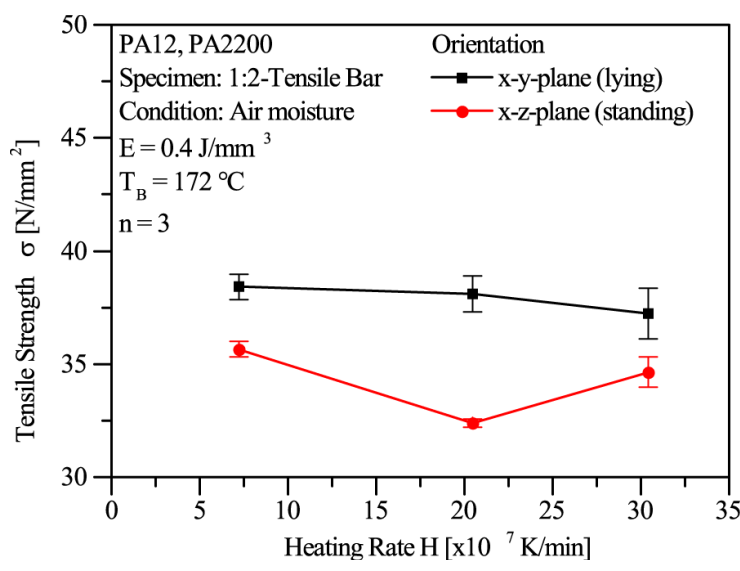

Figure 8. Tensile strength $\sigma$ as function of exposure heating rate

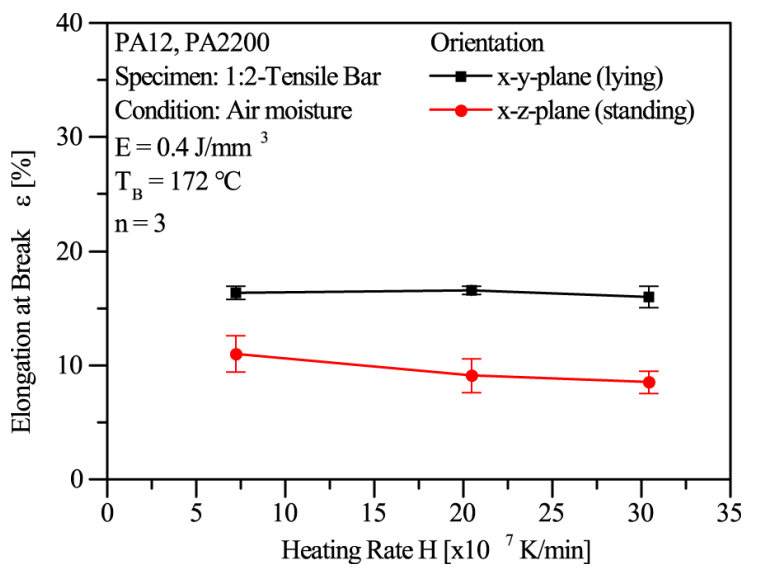

Figure 9. Elongation at break $\varepsilon$ as function of exposure heating rate

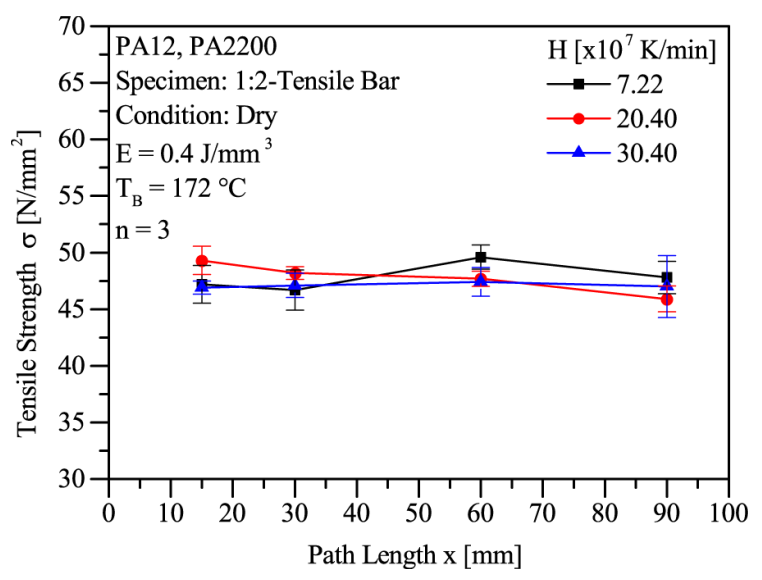

Figure 10. Tensile strength $\sigma$ for varying scanning-path-length $\mathrm{x}$

longer path seem to remain on a constant low level for higher heating rates $\mathrm{H}$. A possible explanation for decreasing elongation at break with longer scanning paths (especially at low heating rates) might be enforced postcondensation-processes. For shorter scanning paths, the cooling times between the first and the second exposure declines (Fig. 12). Hence the surface temperatures increase and post-condensation-processes are performed [9, $12,13]$. Like shown by the authors in prior publications [13] the elongation at break is a sensitive response parameter for changes in post-condensation-processes.

Beneath path length $\mathrm{x}$ the heat flow, caused by the heating rate $H$, influences the mechanical behavior of the tensile 


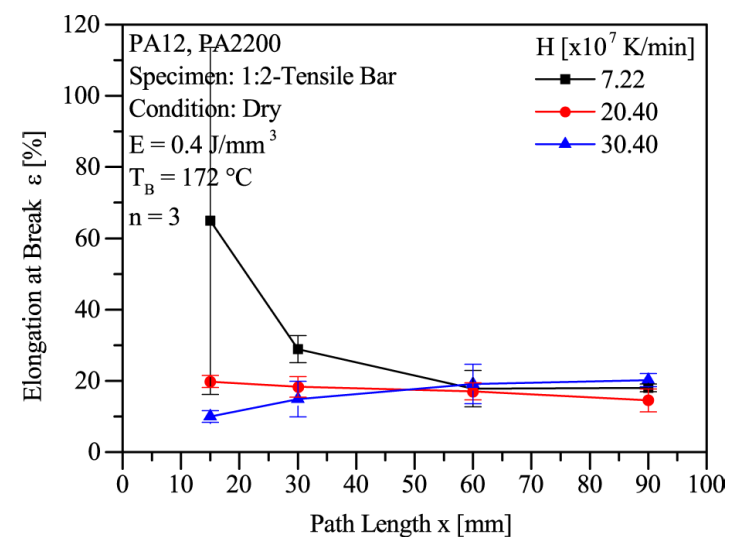

Figure 11. Elongation at break $\varepsilon$ for varying scanning-path-length $\mathrm{x}$

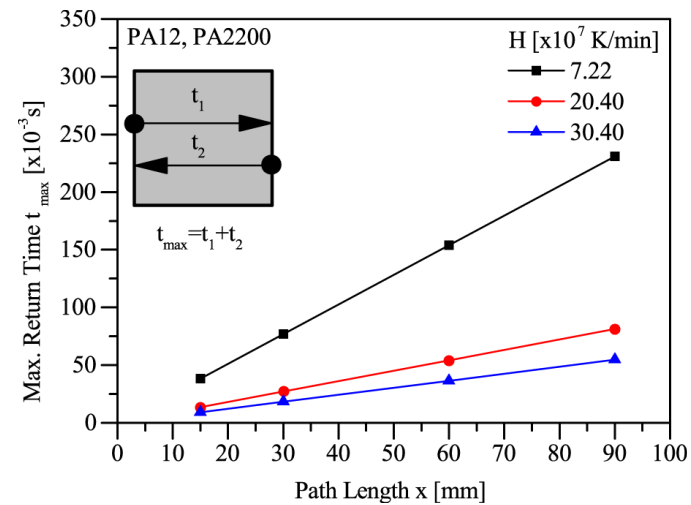

Figure 12. Maximum return time $t_{\max }$ for varying heating rates $\mathrm{H}$

bars. For higher heating rates $H$ the elongation at break is also reduced like for longer scanning paths $\mathrm{x}$. Reasonable for this behavior might be - in addition to postcondensation-processes - decomposition effects within the material. Due to higher heating rates $\mathrm{H}$, temperatures during exposure processes increase as a result of a local heat accumulation between two exposures [9, 12]. The material decomposition changes the mechanical behavior from ductile to a more brittle one. Furthermore, for higher heating rates the effect of reduced elongations for longer paths is reduced, as a result of the high scanning speeds and the along going short times between two exposures. Withal, the changes in mechanical behavior for varying heating rates $\mathrm{H}$ might also be caused by changing part porosity, like investigated by the authors in prior publications [14]. Moreover, mechanical properties are also a function of geometrical part shape [15]. Especially the size of the cross section is affected by varying heating rates according to prior Fig. 6 . The analyzed tensile bars support this estimation. According to Fig. 13 the thickness of the tested tensile bars is primarily a function of the applied heating rate and secondarily of the path length.

Reasonable for this behavior are time dependent effects, which affect heat conduction processes during exposure. For lower heating rates, more time for heat conduction is available. Consequently, process energy is conducted over a larger volume of powder. Owing to this, especially in zones next to the edges of the exposed geometry, along to the heat streams single particles are sticking next to the molten zone. The result is a larger cross section than for higher heating rates.

Like shown in prior results, the mechanical properties of laser molten parts are directly influenced by the length of

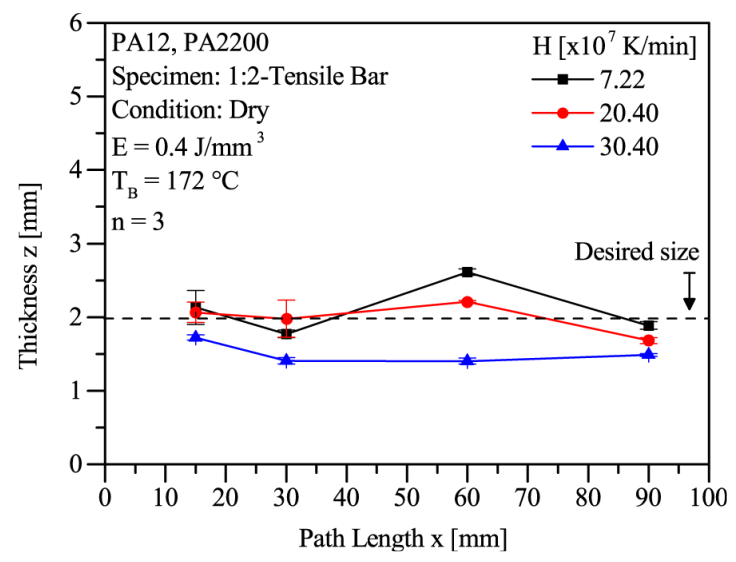

Figure 13. Thickness of tested tensile bars for varying scanning-pathlength $\mathrm{x}$

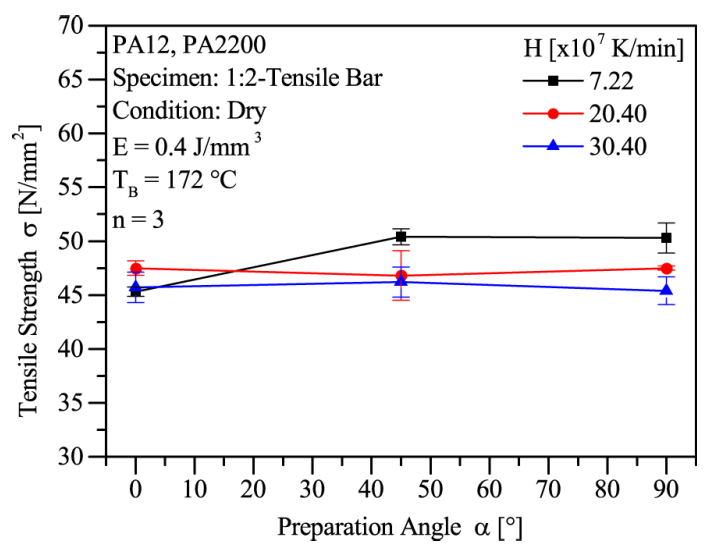

Figure 14. Tensile strength $\sigma$ for varying extraction angles $\alpha$

scanning paths. Reasonable for this fact are the cooling times between two exposures of one single line, due to the hatching principal. Beneath part construction itself, the length of scanning paths is also predicted by orientation of the part within the building chamber. The authors estimate that constant cooling times as a result of constant path lengths cause equal mechanical part properties, although parts are placed differently in $x-y$-plane. For supporting this thesis plates with constant path length have been manufactured with varying $H$. After that the tensile bars are milled out of the plates under different angles $\alpha$ (Fig. 4). Thus varying $\mathrm{x}$ over part geometry at constant cooling times between single paths can be simulated. Fig. 14 shows $\sigma$ for varying angles $\alpha$ and $H$.

Fig. 14 shows clearly that the extraction angle does not seem to influence the tensile strength of the specimen. Despite the extraction angle the heating rate, which was used for manufacturing the plates, influences the tensile strength. An explanation for this behavior might be time dependent heat transfers, like explained in the chapters above. Based on the results of Fig. 14 it is possible to develop new scanning strategies. For example it is possible to place parts in $\mathrm{x}-\mathrm{y}$-plane in different orientations without causing limitations in mechanical behavior as long as the cooling time between single scanning paths is constant (analog to the plate specimen). A possible way to realize this constant cooling time between single paths is a software compensation which adds delay times according to varying path lengths. Additionally constant cooling times between the lines also lead into constant elongations at break over extraction angles, like shown in Fig. 15. 
PAPER

Interaction Between Time DePendent Exposure Strategies And Part Positioning Within SELECTIVE LASER...

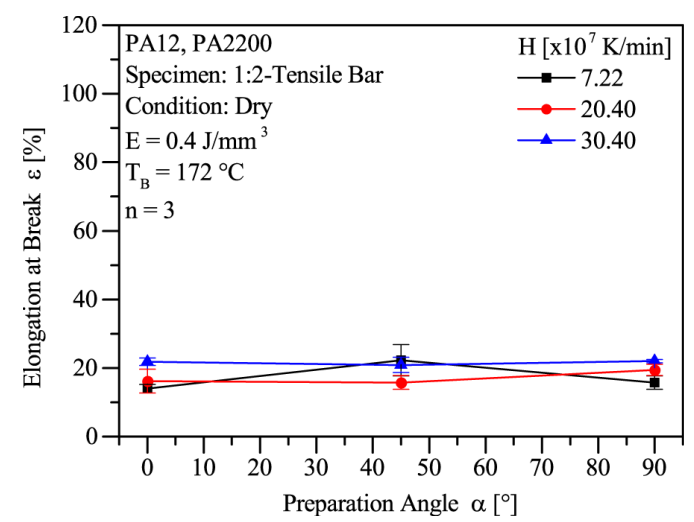

Figure 15. Elongation at break $\varepsilon$ for varying extraction angles $\alpha$

\section{CONCLUSION AND OUTLOOK}

Within the paper the authors discussed time dependent effects on the mechanical part properties according to varying exposure strategies. Therefore plates with varying path lengths have been manufactured. Afterwards tensile bars have been etched out of these plates by milling. Due to this method, disturbing effects like changed surface qualities and along going variations in crack ignition can be minimized. Furthermore, a changed distribution of path lengths over the part geometry can be simulated for constant cooling times between two paths. Based on this design of experiments the authors could show that for an increasing speed of exposure, tensile strength of the bars is marginally affected. The elongation at break instead is decreasing for higher heating rates. Reasonable for this behavior might be material decomposition owing to higher temperatures caused by a heat accumulation for fast exposures. Additionally, for longer scanning paths the cooling times between two single line exposures increase. Based on this, no significant influence on the tensile strength of the bars could be investigated. Nevertheless elongation at break is reduced for longer scanning paths. The authors estimate that changes in post-condensation-processes as a result of changed cooling times between two exposures might be the reason. Considering part positioning in $x-y-$ plane, the authors could show that part orientation might not affect mechanical properties as long as the cooling time between paths is kept constant. Based on those investigations new processing strategies, which consider time dependent effects, can be set up. Exemplary a segmentation of parts' cross sections in segments of constant path length $\mathrm{x}$ is an imaginable processing strategy derived from further described results. Hence path length $\mathrm{x}$ is kept constant independent from the shape of the produced part. Furthermore, a variation of scanning speed and laser power according to path length $\mathrm{x}$ might result in constant return times between hatched paths. Consequently, cooling conditions between paths are proposed to be homogenized.

\section{ACKNOWLEDGMENT}

The authors want to thank the German Research Foundation (DFG) for funding the Collaborative Research Center 814 (CRC 814), sub-project B03.

\section{REFERENCES}

[1] T. Wohlers, Wohlers Report 2011-State of the industry. 2011.

[2] B. Caulfield, P.E. McHugh and S. Lohfeld, "Dependence of mechanical properties of polyamide components on build parame- ters in the SLS process", In: Journal of Materials Processing Technology 182 (2007), S. 477-488. http://dx.doi.org/10.1016/ j.jmatprotec.2006.09.007

[3] J.P. Kruth, B. Vandenbroucke, J. Van Vaerenbergh and P. Mercelis, "Benchmarking of different SLS/SLM processes as rapid manufacturing techniques", Int. Conf. Polymers \& Moulds Innovations, Gent, Belgium, 2005.

[4] D. Rietzel, F. Kühnlein, R. Feulner, G. Hülder, C. Von Wilmowsky, C. Fruth, E. Nkenke and E. Schmachtenberg, "Breaking Material Limitations in Selective Laser Sintering - An Opportunity for Medical Additive Processing", in: S.E.C.o.M. Polymers (Ed.), SPE European Conference on Medical Polymers, Belfast, UK, 2008, pp. 61-65.

[5] W. Hartmann, T. Hausotte, D. Drummer and K. Wudy, „Anforderungen und Randbedingungen für den Einsatz optischer Messsysteme zur In-Line-Prüfung additiv gefertigter Bauteile“, In: RTejournal - Forum für Rapid Technologie 2012 (2012).

[6] B. Wendel, D. Rietzel, F. Kühnlein, R. Feulner, G. Hülder, E. Schmachtenberg, „Additive Processing of Polymers“, In: Macromolecular Materials and Engineering 293 (2008), S. 799-809. http://dx.doi.org/10.1002/mame.200800121

[7] D. Rietzel, M. Drexler, F. Kühnlein and D. Drummer, "Influence of temperature fields on the processing of polymer powders by means of laser and mask sintering technology", Solid Freeform Fabrication (SFF), Austin, Texas, 2011, pp. 252-262.

[8] D. Rietzel, M. Drexler and D. Drummer, „Grundlegende Betrachtungen zur Modellierung transienter thermischer Vorgänge beim selektiven Lasersintern von Thermoplasten“, RTejournal - Forum für Rapid Technologie, Erlangen, 2011.

[9] D. Drummer, M. Drexler and K. Wudy, "Resulting melt-poolshape during selective beam melting of thermoplastics as function of energy input parameters", DDMC, Fraunhofer, Berlin, 2014.

[10] D. Drummer, M. Drexler, K. Wudy and F. Kühnlein, "Effects of powder bulk density on the prorsity of laser molten thermoplastic parts", 29th International Conference of the Polymer Processing Society, Nuremberg, 2013.

[11] D. Drummer, K. Wudy and M. Drexler, „Einfluss der Scangeschwindigkeit auf die Bauteileigenschaften pulver- und strahlbasierter Verfahren“, Rapid.Tech, Erfurt, 2013.

[12] D. Drummer, M. Drexler and K. Wudy, "Derivation of heating rate dependent exposure strategies for the selective laser melting of thermoplastic polymers", 30th International Conference of the Polymer Processing Society, Cleveland, 2014.

[13] K. Wudy, D. Drummer and M. Drexler, "Modelling of the Aging Behavior of Polyamide 12 powder during Laser Melting Process", International Conference of the Polymer Processing Society Cleveland, Ohio, 2014.

[14] D. Drummer, M. Drexler and K. Wudy, "Density of laser molten parts as function of powder coating process during additive manufacturing", 7th World Congress on Particle Technology (WCPT7), Beijing, 2014.

[15] G.W. Ehrenstein, „Polymer-Werkstoffe : Struktur - Eigenschaften - Anwendung“, XVIII, 262 S. Hanser: München [u.a.], 1999. ISBN 3446211616 (kart.)

\section{AUTHORS}

D. Drummer, Head of institute of Polymer Technology, Friedrich-Alexander-University Erlangen-Nürnberg, 91058 Germany (info@lkt.uni-erlangen.de).

M. Drexler, Institute of Polymer Technology, Friedrich-Alexander-University Erlangen-Nürnberg, 91058 Germany (drexler@1kt.uni-erlangen.de).

K. Wudy, Institute of Polymer Technology, Friedrich-Alexander-University Erlangen-Nürnberg, 91058 Germany (wudy@lkt.uni-erlangen.de).

This work was supported by German Research Foundation within the Collaborative Research Center 814, sub-project B03. It is an extended and modified version of a paper presented at the International Conference on Additive Technologies (ICAT2014), held from 15-17 October 2014 in Vienna, Austria. Submitted, 28 November 2014. Published as resubmitted by the authors 10 March 2015 . 